Korean J. Math. 20 (2012), No. 1, pp. 61-75

\title{
VARIOUS FRAMES AND CONNECTIONS
}

\author{
Yong Chan Kim*and Jung Mi Ko
}

\begin{abstract}
We investigate the properties of various frames and connections on partially ordered sets. In particular, we study the relations between various connections and various frames.
\end{abstract}

\section{Introduction}

Pawlak [6] introduced rough set theory to generalize the classical set theory. Rough approximations are defined by a partition of the universe which is corresponding to the equivalence relation about information. It is an important mathematical tool for data analysis and knowledge processing [1-8]. Järvinen et.al.[3] define rough approximations on partially order relations that are not necessarily equivalence relations. Wille [8] introduced the formal concept lattices by allowing some uncertainty in data as examples as Galois, dual Galois, residuated and dual residuated connections. Formal concept analysis is an important mathematical tool for data analysis and knowledge processing [1-7]. Orlowska and Rewitzky [5] investigated the algebraic structures of operators of Galois-style connections.

In this paper, we investigate the properties of various relations, frames and connections on partially ordered sets. In particular, we study the relations between various connections and various frames.

\section{Preliminaries}

Let $X$ be a set. A pair $\left(X, e_{X}\right)$ is called a partially order set (simply,

Received January 3, 2012. Revised February 27, 2012. Accepted March 5, 2012. 2010 Mathematics Subject Classification: 06A06, 06A15, 06B30, 54F05,68U35.

Key words and phrases: Preordered sets, lower and upper rough approximations, Galois, dual Galois, residuated and dual residuated connections and frames.

This work was supported by Research Institute of Natural Science of GangneungWonju National University.

* Corresponding author. 
poset)if $e_{X} \subset X \times X$ is reflexive, transitive and anti-symmetric. Let $\left(X, e_{X}\right)$ be a poset. If we define a relation $(x, y) \in e_{X}^{-1}$ iff $(y, x) \in e_{X}$, then $\left(X, e_{X}^{-1}\right)$ is a poset. We can define a poset $\left(P(X), e_{P(X)}\right)$ where $e_{P(X)} \subset P(X) \times P(X)$ as $(A, B) \in e_{P(X)}$ iff $A \subset B$ for $A, B \in P(X)$.

Definition 2.1. [5] Let $\left(X, e_{X}\right)$ and $\left(Y, e_{Y}\right)$ be posets and $f: X \rightarrow$ $Y$ and $g: Y \rightarrow X$ maps.

(1) $\left(e_{X}, f, g, e_{Y}\right)$ is called a Galois connection if for all $x \in X, y \in Y$, $(y, f(x)) \in e_{Y}$ iff $(x, g(y)) \in e_{X}$.

$(2)\left(e_{X}, f, g, e_{Y}\right)$ is called a dual Galois connection if for all $x \in$ $X, y \in Y,(f(x), y) \in e_{Y}$ iff $(g(y), x) \in e_{X}$.

(3) $\left(e_{X}, f, g, e_{Y}\right)$ is called a residuated connection if for all $x \in X, y \in$ $Y,(f(x), y) \in e_{Y}$ iff $(x, g(y)) \in e_{X}$.

(4) $\left(e_{X}, f, g, e_{Y}\right)$ is called a dual residuated connection if for all $x \in X, y \in Y,(y, f(x)) \in e_{Y}$ iff $(g(y), x) \in e_{X}$.

(5) $f$ is an isotone map if $\left(f\left(x_{1}\right), f\left(x_{2}\right)\right) \in e_{Y}$ for all $\left(x_{1}, x_{2}\right) \in e_{X}$.

(6) $f$ is an antitone map if $\left(f\left(x_{2}\right), f\left(x_{1}\right)\right) \in e_{Y}$ for all $\left(x_{1}, x_{2}\right) \in e_{X}$.

Definition 2.2. [4,5] Let $R \subset X \times Y$ be a relation. For each $B \in$ $P(Y)$, we define operations $[R],[[R]],\langle R\rangle,[\langle R\rangle],[R]^{c},\langle R\rangle^{c}: P(Y) \rightarrow$ $P(X)$ as follows:

$$
\begin{gathered}
{[R](B)=\{x \in X \mid(\forall y \in Y)((x, y) \in R \rightarrow y \in B)\},} \\
{[[R]](B)=\{x \in X \mid(\forall y \in Y)(y \in B \rightarrow(x, y) \in R)\}} \\
\langle R\rangle(B)=\{x \in X \mid(\exists y \in Y)((x, y) \in R \& y \in B)\} \\
{[\langle R\rangle](B)=\left\{x \in X \mid(\exists y \in Y)\left((x, y) \in R^{c} \& y \in B^{c}\right)\right\} .} \\
{[R]^{c}(B)=\left\{x \in X \mid(\forall y \in Y)\left((x, y) \in R \rightarrow y \in B^{c}\right)\right\}} \\
\langle R\rangle^{c}(B)=\left\{x \in X \mid(\exists y \in Y)\left((x, y) \in R \& y \in B^{c}\right)\right\} .
\end{gathered}
$$

For each $R^{-1}=\{(y, x) \in Y \times X \mid(x, y) \in R\}$, we similarly define operations $\left[R^{-1}\right],\left[\left[R^{-1}\right]\right],\left\langle R^{-1}\right\rangle,\left[\left\langle R^{-1}\right\rangle\right],\left[R^{-1}\right]^{c},\left\langle R^{-1}\right\rangle^{c}: P(X) \rightarrow$ $P(Y)$.

Definition 2.3. [4] Let $\left(X, e_{X}\right)$ be a poset. A set $A \in P(X)$ is called an $e_{X}$-upper set if $\left(x \in A \&(x, y) \in e_{X}\right) \rightarrow y \in A$ for $x, y \in X$. 
Theorem 2.4. [4] Let $\left(X, e_{X}\right)$ and $\left(Y, e_{Y}\right)$ be posets and $R \subset X \times Y$ be a relation. For $A \in P(X)$ and $B \in P(Y)$, we define operations as follows:

$$
\begin{gathered}
{\left[e_{X}\right](A)=\left\{x \in X \mid(\forall z \in X)\left((x, z) \in e_{X} \rightarrow z \in A\right)\right\},} \\
\left\langle e_{X}\right\rangle(A)=\left\{x \in X \mid(\exists z \in X)\left((x, z) \in e_{X} \& z \in A\right)\right\}, \\
I(X)=\left\{A \in P(X) \mid\left[e_{X}\right](A)=A\right\}, \\
I(Y)=\left\{B \in P(Y) \mid\left[e_{Y}\right](B)=B\right\},
\end{gathered}
$$

Then we have the following properties:

(1)If $\left(e_{X}\right)_{x}=\left\{z \in X \mid(x, z) \in e_{X}\right\}$ and $\left(e_{X}\right)_{x}^{-1}=\{z \in X \mid(z, x) \in$ $\left.e_{X}\right\}$, then $\left(e_{X}\right)_{x}$ and $\left(\left(e_{X}\right)_{x}^{-1}\right)^{c}$ are $e_{X}$-upper sets.

(2) $\left(e_{X} \circ R\right)^{-1}=R^{-1} \circ e_{X}^{-1}$ and $\left(R \circ e_{Y}\right)^{-1}=e_{Y}^{-1} \circ R^{-1}$.

(3) $e_{X} \circ R \subset R$ iff $e_{X}^{-1} \circ R^{c} \subset R^{c}$.

(4) $R \circ e_{Y}^{-1} \subset R$ iff $R^{c} \circ e_{Y} \subset R^{c}$.

(5) $A$ is an $e_{X}$-upper set iff $\left[e_{X}\right](A)=A$ iff $\left[e_{X}^{-1}\right]\left(A^{c}\right)=A^{c}$ iff $\left\langle e_{X}^{-1}\right\rangle(A)=A$.

(6) If $e_{X} \circ R \subset R$, then $\left(R_{y}^{-1}\right)^{*},[R](B),[R]^{c}(B),[\langle R\rangle](B),\left\langle R^{c}\right\rangle(B)$, $\left\langle R^{c}\right\rangle^{c}(B),\left[\left[R^{c}\right]\right](B) \in I(X)$.

(7) If $e_{X}^{-1} \circ R \subset R$, then $R_{y}^{-1},\langle R\rangle(B),\langle R\rangle^{c}(B),[[R]](B),\left[R^{c}\right](B)$, $\left[R^{c}\right]^{c}(B),\left[\left\langle R^{c}\right\rangle\right](B) \in I(X)$.

(8) If $R \circ e_{Y} \subset R$, then $R_{x},\left\langle R^{-1}\right\rangle(A),\left[\left[R^{-1}\right]\right](A),\left[\left(R^{c}\right)^{-1}\right](A)$, $\left[\left(R^{c}\right)^{-1}\right]^{c}(A),\left\langle R^{-1}\right\rangle^{c}(A) \in I(Y)$.

(9) If $R \circ e_{Y}^{-1} \subset R$, then $R_{x}^{c},\left[R^{-1}\right](A),\left[R^{-1}\right]^{c}(A),\left[\left\langle R^{-1}\right\rangle\right](A)$, $\left\langle\left(R^{c}\right)^{-1}\right\rangle(A),\left\langle\left(R^{c}\right)^{-1}\right\rangle^{c}(A),\left[\left[\left(R^{c}\right)^{-1}\right]\right](A) \in I(Y)$.

Definition $2.5[3,6]$. In above theorem, $\left[e_{X}\right](A)$ and $\left\langle e_{X}\right\rangle(A)$ are called rough lower approximation and rough upper approximation, respectively, for $A \in P(X)$ on a partially ordered set.

If $e_{X}$ is an equivalence relation, $\left[e_{X}\right](A)$ and $\left\langle e_{X}\right\rangle(A)$ are rough lower approximation and rough upper approximation for $A \in P(X)$ in a Pawlak's sense [6]. 


\section{The properties of rough approximations}

Definition $3.1[3,5]$. Let $\left(X, e_{X}\right)$ and $\left(Y, e_{Y}\right)$ be posets, $R \in$ $P(X \times Y)$ and $S \in P(Y \times X)$. A structure $\left(e_{X}, R, S, e_{Y}\right)$ is called:

(1) a Galois frame if $S=R^{-1}$ and $e_{X} \circ R \circ e_{Y}^{-1} \subset R$.

(2) a dual Galois frame if $S=R^{-1}$ and $e_{X}^{-1} \circ R \circ e_{Y} \subset R$.

(3) a residuated frame if $S=R^{-1}$ and $e_{X} \circ R \circ e_{Y} \subset R$.

(4) a dual residuated frame if $S=R^{-1}$ and $e_{X}^{-1} \circ R \circ e_{Y}^{-1} \subset R$.

LEMMA 3.2. Let $\left(X, e_{X}\right)$ and $\left(Y, e_{Y}\right)$ be posets and $f: X \rightarrow Y$ and $g: Y \rightarrow X$ maps.

(1) If $g$ is antitone and define $(x, y) \in R_{1}$ iff $(x, g(y)) \in e_{X}$ (resp. $(x, y) \in R_{2}$ iff $\left.(g(y), x) \in e_{X}\right)$, then $e_{X} \circ R_{1} \circ e_{Y}^{-1} \subset R_{1}$ (resp. $e_{X}^{-1} \circ$ $\left.R_{2} \circ e_{Y} \subset R_{2}\right)$.

(2) If $g$ is isotone and define $(x, y) \in R_{1}$ iff $(x, g(y)) \in e_{X}$ (resp. $(x, y) \in R_{2}$ iff $\left.(g(y), x) \in e_{X}\right)$, then $e_{X} \circ R_{1} \circ e_{Y} \subset R_{1}$ (resp. $e_{X}^{-1} \circ R_{2} \circ$ $\left.e_{Y}^{-1} \subset R_{2}\right)$.

(3) If $f$ is antitone and $(x, y) \in R_{1}$ iff $(y, f(x)) \in e_{Y}$ (resp. $(x, y) \in$ $R_{2}$ iff $\left.(f(x), y) \in e_{Y}\right)$, then $e_{X} \circ R_{1} \circ e_{Y}^{-1} \subset R_{1}$ (resp. $\left.e_{X}^{-1} \circ R_{2} \circ e_{Y} \subset R_{2}\right)$.

(4) If $f$ is isotone and $(x, y) \in R_{1}$ iff $(y, f(x)) \in e_{Y}\left(\operatorname{resp} .(x, y) \in R_{2}\right.$ iff $\left.(f(x), y) \in e_{Y}\right)$, then $e_{X}^{-1} \circ R_{1} \circ e_{Y}^{-1} \subset R_{1}$ (resp. $\left.e_{X} \circ R_{2} \circ e_{Y} \subset R_{2}\right)$.

Proof. (1) Since $g$ is antitone, $\left(y, y_{1}\right) \in e_{Y}$ implies $\left(g\left(y_{1}\right), g(y)\right) \in$ $e_{X}$. Then

$$
\begin{aligned}
& \left(x, x_{1}\right) \in e_{X} \&\left(x_{1}, y_{1}\right) \in R_{1} \&\left(y_{1}, y\right) \in e_{Y}^{-1} \\
& \operatorname{iff}\left(x, x_{1}\right) \in e_{X} \&\left(x_{1}, g\left(y_{1}\right)\right) \in e_{X} \&\left(y, y_{1}\right) \in e_{Y} \\
& (\Rightarrow)\left(x, x_{1}\right) \in e_{X} \&\left(x_{1}, g\left(y_{1}\right)\right) \in e_{X} \&\left(g\left(y_{1}\right), g(y)\right) \in e_{X} \\
& (\Rightarrow)(x, g(y)) \in e_{X} \text { iff }(x, y) \in R
\end{aligned}
$$

Hence $e_{X} \circ R_{1} \circ e_{Y}^{-1} \subset R_{1}$.

Other cases are similarly proved.

Theorem 3.3. Let $\left(X, e_{X}\right)$ and $\left(Y, e_{Y}\right)$ be posets and $f: X \rightarrow Y$ and $g: Y \rightarrow X$ maps.

(1) If $\left(e_{X}, f, g, e_{Y}\right)$ is a Galois connection and define relations $R \in$ $P(X \times Y)$ and $S \in P(Y \times X)$ as

$$
(x, y) \in R \text { iff }(x, g(y)) \in e_{X}, \quad(y, x) \in S \text { iff }(x, y) \in R .
$$


Then $\left(e_{X}, R, S, e_{Y}\right)$ is a Galois frame.

(2) If $\left(e_{X}, f, g, e_{Y}\right)$ is a dual Galois connection and define maps relations $R \in P(X \times Y)$ and $S \in P(Y \times X)$ as

$$
(x, y) \in R \text { iff }(g(y), x) \in e_{X}, \quad(y, x) \in S \text { iff }(x, y) \in R .
$$

Then $\left(e_{X}, R, S, e_{Y}\right)$ is a dual Galois frame.

(3) If $\left(e_{X}, f, g, e_{Y}\right)$ is a residuated connection and define relations $R \in P(X \times Y)$ and $S \in P(Y \times X)$ as

$$
(x, y) \in R \text { iff }(x, g(y)) \in e_{X}, \quad(y, x) \in S \text { iff }(x, y) \in R .
$$

Then $\left(e_{X}, R, S, e_{Y}\right)$ is a residuated frame.

(4) If $\left(e_{X}, f, g, e_{Y}\right)$ is a dual residuated connection and define relations $R \in P(X \times Y)$ and $S \in P(Y \times X)$ as

$$
(x, y) \in R \text { iff }(g(y), x) \in e_{X}, \quad(y, x) \in S \text { iff }(x, y) \in R .
$$

Then $\left(e_{X}, R, S, e_{Y}\right)$ is a dual Galois frame.

Proof. (1) Let $\left(e_{X}, f, g, e_{Y}\right)$ be a Galois connection. If $\left(y_{1}, y_{2}\right) \in e_{Y}$, since $(g(y), g(y)) \in e_{X}$ iff $\left(y, f(g(y)) \in e_{Y}\right.$, we have $\left(y_{1}, y_{2}\right) \in e_{Y}$ and $\left(y_{2}, f\left(g\left(y_{2}\right)\right) \in e_{Y}\right.$ implies $\left(y_{1}, f\left(g\left(y_{2}\right)\right) \in e_{Y}\right.$ iff $\left(g\left(y_{2}\right), g\left(y_{1}\right)\right) \in e_{X}$. Hence $g$ is antitone. By Lemma 3.2(1), $e_{X} \circ R \circ e_{Y}^{-1} \subset R$. Thus $\left(e_{X}, R, S, e_{Y}\right)$ is a Galois frame.

$(2),(3)$ and (4) are similarly proved.

Theorem 3.4. Let $\left(X, e_{X}\right)$ and $\left(Y, e_{Y}\right)$ be posets with $I(X)=$ $\left\{A \in P(X) \mid\left[e_{X}\right](A)=A\right\}$ and $I(Y)=\left\{B \in P(Y) \mid\left[e_{Y}\right](B)=B\right\}$.

(1) If $\left(e_{X}, f, g, e_{Y}\right)$ is a Galois connection and define relations $R \in$ $P(I(X) \times I(Y))$ and $S \in P(I(Y) \times I(X))$ as

$$
\begin{gathered}
(A, B) \in R \text { iff }(\forall x \in X)\left(f(x) \in B \rightarrow x \in A^{c}\right), \\
(B, A) \in S \text { iff }(\forall y \in Y)\left(g(y) \in A \rightarrow y \in B^{c}\right) .
\end{gathered}
$$

Then $\left(e_{I(X)}, R, S, e_{I(Y)}\right)$ is a Galois frame. 
(2) If $\left(e_{X}, f, g, e_{Y}\right)$ is a dual Galois connection and define relations $R \in P(I(X) \times I(Y))$ and $S \in P(I(Y) \times I(X))$ as

$$
\begin{gathered}
(A, B) \in R \text { iff }(\forall x \in X)\left(x \in A^{c} \rightarrow f(x) \in B\right), \\
(B, A) \in S \text { iff }(\forall y \in Y)\left(y \in B^{c} \rightarrow g(y) \in A\right) .
\end{gathered}
$$

Then $\left(e_{I(X)}, R, S, e_{I(Y)}\right)$ is a dual Galois frame.

(3) If $\left(e_{X}, f, g, e_{Y}\right)$ is a residuated connection and define relations $R \in P(I(X) \times I(Y))$ and $S \in P(I(Y) \times I(X))$ as

$$
\begin{gathered}
(A, B) \in R \text { iff }(\forall x \in X)(x \in A \rightarrow f(x) \in B), \\
(B, A) \in S \text { iff }(\forall y \in Y)(y \in B \rightarrow g(y) \in A) .
\end{gathered}
$$

Then $\left(e_{I(X)}, R, S, e_{I(Y)}\right)$ is a residuated frame.

(4) If $\left(e_{X}, f, g, e_{Y}\right)$ is a dual residuated connection and define relations $R \in P(I(X) \times I(Y))$ and $S \in P(I(Y) \times I(X))$ as

$$
\begin{gathered}
(A, B) \in R \text { iff }(\forall x \in X)(f(x) \in B \rightarrow x \in A), \\
(B, A) \in S \text { iff }(\forall y \in Y)(y \in B \rightarrow g(y) \in A) .
\end{gathered}
$$

Then $\left(e_{I(X)}, R, S, e_{I(Y)}\right)$ is a dual residuated frame.

Proof. (1) We have $\left(A, A^{\prime}\right) \in e_{P(X)} \&\left(A^{\prime}, B^{\prime}\right) \in R \&\left(B^{\prime}, B\right) \in$ $e_{P(Y)}^{-1} \subset(A, B) \in R$ from:

$$
\begin{aligned}
& \left(f(x) \in B \rightarrow f(x) \in B^{\prime}\right) \&\left(f(x) \in B^{\prime} \rightarrow x \in A^{\prime c}\right) \&\left(x \in A^{\prime c} \rightarrow x \in A^{c}\right) \\
& \rightarrow\left(f(x) \in B \rightarrow x \in A^{c}\right)
\end{aligned}
$$

Hence $e_{P(X)} \circ R \circ e_{P(Y)}^{-1} \subset R$.

For $A \in I(X), B \in I(Y)$ and a Galois connection $\left(e_{X}, f, g, e_{Y}\right)$, since $x \in A$ iff $x \in A$ and $(x, g(f(x))) \in e_{X}$ implies $g(f(x)) \in A$ and $y \in B$ iff $y \in B$ and $(y, f(g(y))) \in e_{Y}$ implies $f(g(y)) \in B$, we have $R^{-1}=S$ from:

$$
\begin{aligned}
(A, B) \in R \text { iff }(\forall x \in X)\left(\left(f(x) \in B \rightarrow x \in A^{c}\right)\right. \\
\quad(\Rightarrow)(\forall y \in Y)\left(f(g(y)) \in B \rightarrow g(y) \in A^{c}\right) \\
\quad(\Rightarrow)(\forall y \in Y)\left(y \in B \rightarrow g(y) \in A^{c}\right) \\
\quad(\Rightarrow)(\forall y \in Y)\left(g(y) \in A \rightarrow y \in B^{c}\right) \\
\quad(\Rightarrow)(B, A) \in S
\end{aligned}
$$




$$
\begin{aligned}
(B, A) \in S \text { iff }(\forall y \in Y)\left(g(y) \in A \rightarrow y \in B^{c}\right) \\
\\
\quad \Rightarrow)(\forall x \in X)\left(g(f(x)) \in A \rightarrow f(x) \in B^{c}\right) \\
\quad(\Rightarrow)(\forall x \in X)\left(x \in A \rightarrow f(x) \in B^{c}\right) \\
\quad(\Rightarrow)(\forall x \in X)\left(f(x) \in B \rightarrow x \in A^{c}\right) \\
\quad(\Rightarrow)(A, B) \in R .
\end{aligned}
$$

(2) We have $\left(A, A^{\prime}\right) \in e_{P(X)}^{-1} \&\left(A^{\prime}, B^{\prime}\right) \in R \&\left(B^{\prime}, B\right) \in e_{P(Y)} \subset$ $(A, B) \in R$ implies $e_{P(X)}^{-1} \circ R \circ e_{P(Y)} \subset R$ from:

$\left(x \in A^{\prime} \rightarrow x \in A\right) \&\left(x \in\left(A^{\prime}\right)^{c} \rightarrow f(x) \in B^{\prime}\right) \&\left(f(x) \in B^{\prime} \rightarrow f(x) \in B\right)$

$\rightarrow\left(x \in A^{c} \rightarrow f(x) \in B\right)$

Since $f(g(y)) \in B \&(f(g(y)), y) \in e_{Y}$ implies $y \in B$, we have $R \subset S^{-1}$ from:

$$
\begin{aligned}
(A, B) \in R & \text { iff }(\forall x \in X)\left(x \in A^{c} \rightarrow f(x) \in B\right) \\
& (\Rightarrow)(\forall y \in Y)\left(g(y) \in A^{c} \rightarrow f(g(y)) \in B\right) \\
& (\Rightarrow)(\forall y \in Y)\left(g(y) \in A^{c} \rightarrow y \in B\right) \\
& (\Rightarrow)(\forall y \in Y)\left(y \in B^{c} \rightarrow g(y) \in A\right) \\
& (\Rightarrow)(B, A) \in S,
\end{aligned}
$$

Similarly, $S \subset R^{-1}$.

(3) For $A, B \in I(X)$, since $f(g(y)) \in B \&(f(g(y)), y) \in e_{Y} \rightarrow x \in$ $B$ and $x \in A \&(x, g(f(x))) \in e_{X} \rightarrow(g(f(x))) \in A$, we have

$$
\begin{aligned}
(A, B) \in R & \text { iff }(\forall x \in X)(x \in A \rightarrow f(x) \in B) \\
& \Rightarrow)(\forall y \in Y)(g(y) \in A \rightarrow f(g(y)) \in B) \\
& (\Rightarrow)(\forall y \in Y)(g(y) \in A \rightarrow y \in B) \\
& (\Rightarrow)(B, A) \in S, \\
(B, A) \in S & \text { iff }(\forall y \in Y)(g(y) \in A \rightarrow y \in B) \\
& (\Rightarrow)(\forall x \in X)(g(f(x)) \in A \rightarrow f(x) \in B) \\
& (\Rightarrow)(\forall x \in X)(x \in A \rightarrow f(x) \in B) \\
& (\Rightarrow)(A, B) \in R .
\end{aligned}
$$


(4) For $A \in I(X)$, since $g(f(x)) \in A \&(g(f(x)), x) \in e_{X} \rightarrow x \in A$ and $y \in B \&(y, f(g(y))) \in e_{Y} \rightarrow f(g(y)) \in B$, we have

$$
\begin{aligned}
(A, B) \in R & \text { iff }(\forall x \in X)((f(x) \in B \rightarrow x \in A) \\
& (\Rightarrow)(\forall y \in Y)(f(g(y)) \in B \rightarrow g(y) \in A) \\
& (\Rightarrow)(\forall y \in Y)(y \in B \rightarrow g(y) \in A) \\
& (\Rightarrow)(B, A) \in S, \\
(B, A) \in S \text { iff }(\forall y \in Y)(y \in B \rightarrow g(y) \in A) & (\Rightarrow)(\forall x \in X)(f(x) \in B \rightarrow g(f(x)) \in A) \\
& (\Rightarrow)(\forall x \in X)(f(x) \in B \rightarrow x \in A) \\
& (\Rightarrow)(A, B) \in R .
\end{aligned}
$$

TheOREM 3.5. Let $\left(e_{X}, R, S, e_{Y}\right)$ be a Galois frame.

(1) If we define $\left[R^{-1}\right]^{c}: I(X) \rightarrow I(Y)$ and $\left[S^{-1}\right]^{c}: I(Y) \rightarrow I(X)$ as

$$
\begin{gathered}
y \in\left[R^{-1}\right]^{c}(A) \text { iff }(\forall x \in X)\left((x, y) \in R \rightarrow x \in A^{c}\right) \\
x \in\left[S^{-1}\right]^{c}(B) \text { iff }(\forall y \in Y)\left((y, x) \in S \rightarrow y \in B^{c}\right)
\end{gathered}
$$

Then $\left(e_{I(X)},\left[R^{-1}\right]^{c},\left[S^{-1}\right]^{c}, e_{I(Y)}\right)$ is a Galois connection.

(2) If we define $\left[\left\langle R^{-1}\right\rangle\right]: I(X) \rightarrow I(Y)$ and $\left[\left\langle S^{-1}\right\rangle\right]: I(Y) \rightarrow I(X)$ as

$$
\begin{aligned}
& y \in\left[\left\langle R^{-1}\right\rangle\right](A) \text { iff }(\exists x \in X)\left(x \in A^{c} \&(x, y) \in R^{c}\right) \\
& x \in\left[\left\langle S^{-1}\right\rangle\right](B) \text { iff }(\exists y \in Y)\left(y \in B^{c} \&(y, x) \in S^{c}\right) .
\end{aligned}
$$

Then $\left(e_{I(X)},\left[\left\langle R^{-1}\right\rangle\right],\left[\left\langle S^{-1}\right\rangle\right], e_{I(Y)}\right)$ is a dual Galois connection.

Proof. (1) Since $R \circ e_{Y}^{-1} \subset e_{X} \circ R \circ e_{Y}^{-1} \subset R$, by Theorem 2.4 (9), $\left[R^{-1}\right]^{c}(A) \in I(Y)$. Since $\left(e_{X} \circ R \circ e_{Y}^{-1}\right)^{-1}=e_{Y} \circ S \circ e_{X}^{-1} \subset S$, by Theorem $2.4(9),\left[S^{-1}\right]^{c}(B) \in I(X)$. Hence $\left[R^{-1}\right]^{c}$ and $\left[S^{-1}\right]^{c}$ is well 
defined. Moreover, $\left(e_{I(X)},\left[R^{-1}\right]^{c},\left[S^{-1}\right]^{c}, e_{I(Y)}\right)$ is a Galois connection from

$$
\begin{aligned}
& B \subset\left[R^{-1}\right]^{c}(A) \text { iff } \vdash(\forall y \in Y)\left(y \in B \rightarrow y \in\left[R^{-1}\right]^{c}(A)\right) \\
& \text { iff } \vdash(\forall y \in Y)\left(y \in B \rightarrow(\forall x \in X)\left((x, y) \in R \rightarrow x \in A^{c}\right)\right) \\
& \text { iff } \vdash(\forall y \in Y)(\forall x \in X)\left(y \in B \rightarrow\left(x \in A \rightarrow(x, y) \in R^{c}\right)\right) \\
& \text { iff } \vdash(\forall y \in Y)(\forall x \in X)\left(x \in A \rightarrow\left(y \in B \rightarrow(x, y) \in R^{c}\right)\right) \\
& \text { iff } \vdash(\forall y \in Y)(\forall x \in X)\left(x \in A \rightarrow\left((x, y) \in R \rightarrow y \in B^{c}\right)\right) \\
& \text { iff } \vdash(\forall x \in X)\left(x \in A \rightarrow(\forall y \in Y)\left((x, y) \in R \rightarrow y \in B^{c}\right)\right) \\
& \text { iff } \vdash(\forall x \in X)\left(x \in A \rightarrow x \in\left[S^{-1}\right]^{c}(B)\right) \\
& \text { iff } A \subset\left[S^{-1}\right]^{c}(B) .
\end{aligned}
$$

(2) Since $R \circ e_{Y}^{-1} \subset e_{X} \circ R \circ e_{Y}^{-1} \subset R$, by Theorem 2.4 (9), then $\left[\left\langle R^{-1}\right\rangle\right](A) \in I(Y)$. Since $\left(e_{X} \circ R \circ e_{Y}^{-1}\right)^{-1}=e_{Y} \circ S \circ e_{X}^{-1} \subset S$, by Theorem $2.4(9),\left[\left\langle S^{-1}\right\rangle\right](B) \in I(X)$. Hence $\left[\left\langle R^{-1}\right\rangle\right]$ and $\left[\left\langle S^{-1}\right\rangle\right]$ is well defined. Moreover, $\left(e_{I(X)},\left[\left\langle R^{-1}\right\rangle\right],\left[\left\langle S^{-1}\right\rangle\right], e_{I(Y)}\right)$ is a dual Galois connection from

$$
\begin{aligned}
& {\left[\left\langle R^{-1}\right\rangle\right](A) \subset B \text { iff } \vdash(\forall y \in Y)\left(y \in\left[\left\langle R^{-1}\right\rangle\right](A) \rightarrow y \in B\right)} \\
& \text { iff } \vdash(\forall y \in Y)\left((\exists x \in X)\left(x \in A^{c} \&(x, y) \in R^{c}\right) \rightarrow y \in B\right) \\
& \text { iff } \vdash(\forall y \in Y)(\forall x \in X)\left((x, y) \in R^{c}\right) \rightarrow\left(x \in A^{c} \rightarrow y \in B\right) \\
& \text { iff } \vdash(\forall y \in Y)(\forall x \in X)\left((x, y) \in R^{c}\right) \rightarrow\left(y \in B^{c} \rightarrow x \in A\right) \\
& \text { iff } \vdash(\forall x \in X)\left((\exists y \in Y)\left(y \in B^{c} \&(x, y) \in R^{c}\right) \rightarrow x \in A\right) \\
& \text { iff } \vdash(\forall x \in X)\left(x \in\left[\left\langle S^{-1}\right\rangle\right](B) \rightarrow x \in A\right) \\
& \text { iff }\left[\left\langle S^{-1}\right\rangle\right](B) \subset A .
\end{aligned}
$$

Theorem 3.6. Let $\left(e_{X}, R, S, e_{Y}\right)$ be a dual Galois frame.

(1) If we define $\left\langle R^{-1}\right\rangle^{c}: I(X) \rightarrow I(Y)$ and $\left\langle S^{-1}\right\rangle^{c}: I(Y) \rightarrow I(X)$ as

$$
\begin{aligned}
& y \in\left\langle R^{-1}\right\rangle^{c}(A) \text { iff }(\exists x \in X)\left(x \in A^{c} \&(x, y) \in R\right) \\
& x \in\left\langle S^{-1}\right\rangle^{c}(B) \text { iff }(\exists y \in Y)\left(y \in B^{c} \&(y, x) \in S\right) .
\end{aligned}
$$


Then $\left(e_{I(X)},\left\langle R^{-1}\right\rangle^{c},\left\langle S^{-1}\right\rangle^{c}, e_{I(Y)}\right)$ is a dual Galois connection.

(2) If we define $\left[\left[R^{-1}\right]\right]: I(X) \rightarrow I(Y)$ and $\left[\left[S^{-1}\right]\right]: I(Y) \rightarrow I(X)$ as

$$
\begin{aligned}
& y \in\left[\left[R^{-1}\right]\right](A) \text { iff }(\forall x \in X)(x \in A \rightarrow(x, y) \in R) \\
& x \in\left[\left[S^{-1}\right]\right](B) \text { iff }(\forall y \in Y)(y \in B \rightarrow(y, x) \in S) .
\end{aligned}
$$

Then $\left(e_{I(X)},\left[\left[R^{-1}\right]\right],\left[\left[S^{-1}\right]\right], e_{I(Y)}\right)$ is a Galois connection.

Proof. (1) Since $R \circ e_{Y} \subset e_{X}^{-1} \circ R \circ e_{Y} \subset R$, by Theorem 2.4 (8), $\left\langle R^{-1}\right\rangle^{c}(A) \in I(Y)$. Since $\left(e_{X}^{-1} \circ R \circ e_{Y}\right)^{-1}=e_{Y}^{-1} \circ S \circ e_{X} \subset S$, by Theorem $2.4(8),\left\langle S^{-1}\right\rangle^{c}(B) \in I(X)$. Hence $\left\langle R^{-1}\right\rangle^{c}$ and $\left\langle S^{-1}\right\rangle^{c}$ is well defined. Moreover, $\left(e_{I(X)},\left\langle R^{-1}\right\rangle^{c},\left\langle S^{-1}\right\rangle^{c}, e_{I(Y)}\right)$ is a dual Galois connection from

$$
\begin{aligned}
& \left\langle R^{-1}\right\rangle^{c}(A) \subset B \text { iff } \vdash(\forall y \in Y)\left(y \in\left\langle R^{-1}\right\rangle^{c}(A) \rightarrow y \in B\right) \\
& \text { iff } \vdash(\forall y \in Y)\left((\exists x \in X)\left(x \in A^{c} \&(x, y) \in R \rightarrow y \in B\right)\right) \\
& \text { iff } \vdash(\forall y \in Y)(\forall x \in X)\left((x, y) \in R \rightarrow\left(x \in A^{c} \rightarrow y \in B\right)\right) \\
& \text { iff } \vdash(\forall y \in Y)(\forall x \in X)\left((x, y) \in R \rightarrow\left(y \in B^{c} \rightarrow x \in A\right)\right) \\
& \text { iff } \vdash(\forall x \in X)\left((\exists y \in Y)\left(y \in B^{c} \&(x, y) \in R\right) \rightarrow x \in A\right) \\
& \text { iff } \vdash(\forall x \in X)\left(x \in\left\langle S^{-1}\right\rangle^{c}(B) \rightarrow x \in A\right) \\
& \text { iff }\left\langle S^{-1}\right\rangle^{c}(B) \subset A .
\end{aligned}
$$

(2) Since $R \circ e_{Y} \subset e_{X}^{-1} \circ R \circ e_{Y} \subset R$, by Theorem $2.4(8)$, [[ $\left.\left.R^{-1}\right]\right](A) \in$ $I(X)$. Since $S \circ e_{X} \subset R$, by Theorem $2.4(8)$, [[ $\left.\left.S^{-1}\right]\right](B) \in I(X)$. Then $\left(e_{I(X)},\left[\left[R^{-1}\right]\right],\left[\left[S^{-1}\right]\right], e_{I(Y)}\right)$ is a Galois connection from

$$
\begin{aligned}
& B \subset\left[\left[R^{-1}\right]\right](A) \text { iff } \vdash(\forall y \in Y)\left(y \in B \rightarrow y \in\left[\left[R^{-1}\right]\right](A)\right) \\
& \text { iff } \vdash(\forall y \in Y)(y \in B \rightarrow(\forall x \in X)(x \in A \rightarrow(x, y) \in R)) \\
& \text { iff } \vdash(\forall y \in Y)(\forall x \in X)(y \in B \rightarrow(x \in A \rightarrow(x, y) \in R)) \\
& \text { iff } \vdash(\forall y \in Y)(\forall x \in X)(x \in A \rightarrow(y \in B \rightarrow(x, y) \in R)) \\
& \text { iff } \vdash(\forall x \in X)\left(x \in A \rightarrow x \in\left[\left[S^{-1}\right]\right](B)\right)
\end{aligned}
$$$$
\text { iff } A \subset\left[\left[S^{-1}\right]\right](B) \text {. }
$$ 
TheOREM 3.7. Let $\left(e_{X}, R, S, e_{Y}\right)$ be a residuated frame.

(1) If we define $\left\langle R^{-1}\right\rangle: I(X) \rightarrow I(Y)$ and $\left[S^{-1}\right]: I(Y) \rightarrow I(X)$ as

$$
\begin{aligned}
& y \in\left\langle R^{-1}\right\rangle(A) \text { iff }(\exists x \in X)((x, y) \in R \& x \in A) \\
& x \in\left[S^{-1}\right](B) \text { iff }(\forall y \in Y)((y, x) \in S \rightarrow y \in B)
\end{aligned}
$$

Then $\left(e_{I(X)},\left\langle R^{-1}\right\rangle,\left[S^{-1}\right], e_{I(Y)}\right)$ is a residuated connection.

(2) If we define $\left[\left(R^{c}\right)^{-1}\right]: I(X) \rightarrow I(Y)$ and $\left\langle\left(S^{c}\right)^{-1}: I(Y) \rightarrow I(X)\right.$ as

$$
\begin{aligned}
& y \in\left[\left(R^{c}\right)^{-1}\right](A) \text { iff }(\forall x \in X)\left((x, y) \in R^{c} \rightarrow x \in A\right) \\
& x \in\left\langle\left(S^{c}\right)^{-1}\right\rangle(B) \text { iff }(\exists y \in Y)\left(y \in B \&(y, x) \in S^{c}\right) .
\end{aligned}
$$

Then $\left(e_{I(X)},\left[\left(R^{c}\right)^{-1}\right],\left\langle\left(S^{c}\right)^{-1}\right\rangle, e_{I(Y)}\right)$ is a dual residuated connection.

Proof. (1) Since $R \circ e_{Y} \subset e_{X} \circ R \circ e_{Y} \subset R$, by Theorem 2.4 (8), $\left\langle R^{-1}\right\rangle(A) \in I(Y)$. Since $S \circ e_{X}^{-1} \subset\left(e_{X} \circ R \circ e_{Y}\right)^{-1}=e_{Y}^{-1} \circ S \circ e_{X}^{-1} \subset$ $S$, by Theorem $2.4(9),\left[S^{-1}\right](B) \in I(X)$. Hence $\left\langle R^{-1}\right\rangle$ and $\left[S^{-1}\right]$ is well defined. Moreover, $\left(e_{I(X)},\left\langle R^{-1}\right\rangle,\left[S^{-1}\right], e_{I(Y)}\right)$ is a residuated connection from

$$
\begin{aligned}
& \left\langle R^{-1}\right\rangle(A) \subset B \text { iff } \vdash(\forall y \in Y)\left(y \in\left\langle R^{-1}\right\rangle(A) \rightarrow y \in B\right) \\
& \text { iff } \vdash(\forall y \in Y)((\exists x \in X)(x \in A \&(x, y) \in R) \rightarrow y \in B) \\
& \text { iff } \vdash(\forall y \in Y)(\forall x \in X)(x \in A \rightarrow((x, y) \in R \rightarrow y \in B)) \\
& \text { iff } \vdash(\forall x \in X)(x \in A \rightarrow(\forall y \in Y)((x, y) \in R \rightarrow y \in B)) \\
& \text { iff } A \subset\left[S^{-1}\right](B) .
\end{aligned}
$$

(2) Since $R \circ e_{Y} \subset e_{X} \circ R \circ e_{Y} \subset R$ and $R \circ e_{Y} \subset R$ iff $R^{c} \circ e_{Y}^{-1} \subset R^{c}$, by Theorem $2.4(8),\left[\left(R^{c}\right)^{-1}\right](A) \in I(Y)$. Since $S \circ e_{X}^{-1} \subset\left(e_{X} \circ R \circ e_{Y}\right)^{-1}=$ $e_{Y}^{-1} \circ S \circ e_{X}^{-1} \subset S$, by Theorem $2.4(9),\left\langle\left(S^{c}\right)^{-1}\right\rangle(B) \in I(X)$. So, $\left[\left(R^{c}\right)^{-1}\right]$ and $\left\langle\left(S^{c}\right)^{-1}\right\rangle$ is well defined. Thus, $\left(e_{I(X)},\left\langle R^{-1}\right\rangle,\left\langle\left(S^{c}\right)^{-1}\right\rangle, e_{I(Y)}\right)$ is a dual residuated connection from

$$
\begin{aligned}
& B \subset\left[\left(R^{c}\right)^{-1}\right](A) \text { iff } \vdash(\forall y \in Y)\left(y \in B \rightarrow y \in\left[\left(R^{c}\right)^{-1}\right](A)\right) \\
& \text { iff } \vdash(\forall y \in Y)\left(y \in B \rightarrow(\forall x \in X)\left((x, y) \in R^{c} \rightarrow x \in A\right)\right. \\
& \text { iff } \vdash(\forall y \in Y)(\forall x \in X)\left(y \in B \rightarrow\left((x, y) \in R^{c} \rightarrow x \in A\right)\right. \\
& \text { iff } \vdash(\forall y \in Y)(\forall x \in X)\left(\left(y \in B \&(x, y) \in R^{c}\right) \rightarrow x \in A\right) \\
& \text { iff } \vdash(\forall x \in X)\left((\exists y \in Y)\left(y \in B \&(x, y) \in R^{c}\right) \rightarrow x \in A\right) \\
& \text { iff }\left\langle\left(S^{c}\right)^{-1}\right\rangle(B) \subset A .
\end{aligned}
$$


Theorem 3.8. Let $\left(e_{X}, R, S, e_{Y}\right)$ be a dual residuated frame.

(1) If we define $\left\langle\left(R^{c}\right)^{-1}\right\rangle: I(X) \rightarrow I(Y)$ and $\left[\left(S^{c}\right)^{-1}\right]: I(Y) \rightarrow I(X)$ as

$$
\begin{gathered}
y \in\left\langle\left(R^{c}\right)^{-1}\right\rangle(A) \text { iff }(\exists x \in X)\left((x, y) \in R^{c} \& x \in A\right) \\
x \in\left[\left(S^{c}\right)^{-1}\right](B) \text { iff }(\forall y \in Y)\left((y, x) \in S^{c} \rightarrow y \in B\right)
\end{gathered}
$$

Then $\left(e_{I(X)},\left\langle\left(R^{c}\right)^{-1}\right\rangle,\left[\left(S^{c}\right)^{-1}\right], e_{I(Y)}\right)$ is a residuated connection.

(2) If we define $\left[R^{-1}\right]: I(X) \rightarrow I(Y)$ and $\left\langle S^{-1}\right\rangle: I(Y) \rightarrow I(X)$ as

$$
\begin{aligned}
& y \in\left[R^{-1}\right](A) \text { iff }(\forall x \in X)((x, y) \in R \rightarrow x \in A) \\
& x \in\left\langle S^{-1}\right\rangle(B) \text { iff }(\exists y \in Y)((y, x) \in S \& y \in B)
\end{aligned}
$$

Then $\left(e_{I(X)},\left[R^{-1}\right],\left\langle S^{-1}\right\rangle, e_{I(Y)}\right)$ is a dual residuated connection.

Proof. (1) Since $R \circ e_{Y}^{-1} \subset e_{X}^{-1} \circ R \circ e_{Y}^{-1} \subset R$ and $R \circ e_{Y}^{-1} \subset R$ iff $R^{c} \circ e_{Y} \subset R^{c}$, by Theorem 2.4(9), $\left\langle\left(R^{c}\right)^{-1}\right\rangle(A) \in I(Y)$. Since $S \circ e_{X} \subset\left(e_{X}^{-1} \circ R \circ e_{Y}^{-1}\right)^{-1}=e_{Y} \circ S \circ e_{X} \subset S$, by Theorem 2.4 (8), $\left[\left(S^{c}\right)^{-1}\right](B) \in I(X)$. Hence $\left\langle\left(R^{c}\right)^{-1}\right\rangle$ and $\left[\left(S^{c}\right)^{-1}\right]$ is well defined. Then $\left(e_{I(X)},\left\langle\left(R^{c}\right)^{-1}\right\rangle,\left[\left(S^{c}\right)^{-1}\right], e_{I(Y)}\right)$ is a residuated connection from:

$$
\begin{aligned}
& \left\langle\left(R^{c}\right)^{-1}\right\rangle(A) \subset B \text { iff } \vdash(\forall y \in Y)\left(y \in\left\langle\left(R^{c}\right)^{-1}\right\rangle(A)(A) \rightarrow y \in B\right) \\
& \text { iff } \vdash(\forall y \in Y)\left((\exists x \in X)\left(x \in A \&(x, y) \in R^{c}\right) \rightarrow y \in B\right) \\
& \text { iff } \vdash(\forall y \in Y)(\forall x \in X)\left(x \in A \rightarrow\left((x, y) \in R^{c} \rightarrow y \in B\right)\right) \\
& \text { iff } \vdash(\forall y \in Y)(\forall x \in X)\left(x \in A \rightarrow(\forall y \in Y)\left((x, y) \in R^{c} \rightarrow y \in B\right)\right) \\
& \text { iff } A \subset\left[\left(S^{c}\right)^{-1}\right](B) .
\end{aligned}
$$

(2) Since $R \circ e_{Y}^{-1} \subset e_{X}^{-1} \circ R \circ e_{Y}^{-1} \subset R$, by Theorem $2.4(9),\left[R^{-1}\right](A) \in$ $I(Y)$. Since $S \circ e_{X} \subset\left(e_{X}^{-1} \circ R \circ e_{Y}^{-1}\right)^{-1}=e_{Y} \circ S \circ e_{X} \subset S$, by Theorem $2.4(8),\left\langle S^{-1}\right\rangle(B) \in I(X)$. Hence $\left[R^{-1}\right]$ and $\left\langle S^{-1}\right\rangle$ is well defined. We similarly proved as in (1). 
ExAmple 3.9. Let $\left(X=\{a, b, c, d\}, e_{X}\right)$ and $\left(Y=\{x, y, z\}, e_{Y}\right)$ be posets with

$$
\begin{gathered}
e_{X}=\{(a, a),(a, b),(a, c),(a, d),(b, b),(b, d),(c, c),(c, d),(d, d)\} \\
e_{Y}=\{(x, x),(x, y),(x, z),(y, y),(z, y),(z, z)\}
\end{gathered}
$$

Put a relation $R \subset X \times Y$ as

$$
R=\{(a, x),(b, x),(b, y),(b, z),(c, x),(c, z),(d, x),(d, y),(d, z)\} .
$$

Then $\left(e_{X}\right)_{a}=\{a, b, c, d\},\left(e_{X}\right)_{b}=\{b, d\},\left(e_{X}\right)_{c}=\{c, d\},\left(e_{X}\right)_{d}=\{d\}$ are $e_{X}$-upper sets. Since $(a, b) \in e_{X} \&(b, y) \in R$, but $(a, y) \notin R$. Hence $e_{X} \circ R \not \subset R$. Since $e_{X}^{-1} \circ R=R, R_{x}^{-1}=\{a, b, c, d\}, R_{y}^{-1}=$ $\{b, d\}, R_{z}^{-1}=\{b, c, d\}$ are $e_{X}$-upper sets. Since $R \circ e_{Y}^{-1}=R, R_{a}^{c}=$ $\{y, z\}, R_{b}^{c}=\emptyset, R_{c}^{c}=\{y\}, R_{d}^{c}=\emptyset$ are $e_{Y}$-upper sets. Since $R \circ e_{Y} \not \subset R$, $R_{a}=\{x\}$ is not an $e_{Y}$-upper sets because $x \in R_{a} \&(x, y) \in e_{Y}$, but $y \notin R_{a}$.

Furthermore, $I(X)=\{\emptyset, X,\{d\},\{b, d\},\{c, d\},\{b, c, d\}\}$ and $I(Y)=$ $\{\emptyset, Y,\{y\},\{y, z\}\}$.

(1) We obtain $\left\langle\left(R^{c}\right)^{-1}\right\rangle: I(X) \rightarrow I(Y)$ and $\left[\left(S^{c}\right)^{-1}\right]: I(Y) \rightarrow I(X)$ as

$$
\begin{gathered}
\left\langle\left(R^{c}\right)^{-1}\right\rangle(A)= \begin{cases}\{y, z\} & \text { if } A=X, \\
\{y\} & \text { if } A \in\{\{b, c, d\},\{c, d\}\} \\
\emptyset & \text { if } A \in\{\emptyset,\{d\},\{b, d\}\}\end{cases} \\
{\left[\left(S^{c}\right)^{-1}\right](B)= \begin{cases}\{b, d\} & \text { if } B=\emptyset, \\
\{b, c, d\} & \text { if } B=\{y\} \\
X & \text { if } B \in\{Y,\{y, z\}\}\end{cases} }
\end{gathered}
$$

Then $\left(e_{I(X)},\left\langle\left(R^{c}\right)^{-1}\right\rangle,\left[\left(S^{c}\right)^{-1}\right], e_{I(Y)}\right)$ is a residuated connection.

(2) We obtain $\left[R^{-1}\right]: I(X) \rightarrow I(Y)$ and $\left\langle S^{-1}\right\rangle: I(Y) \rightarrow I(X)$ as

$$
\left[R^{-1}\right](A)= \begin{cases}Y & \text { if } A=X, \\ \{y, z\} & \text { if } A=\{b, c, d\}, \\ \{y\} & \text { if } A \in\{\{b, d\},\{a, b, d\}\} \\ \emptyset & \text { otherwise }\end{cases}
$$




$$
\left\langle S^{-1}\right\rangle(B)= \begin{cases}X & \text { if } x \in B \\ \{b, c, d\} & \text { if } B \in\{\{y, z\},\{z\}\}, \\ \{b, d\} & \text { if } B=\{y\} \\ \emptyset & \text { if } B=\emptyset .\end{cases}
$$

Then $\left(e_{I(X)},\left[R^{-1}\right],\left\langle S^{-1}\right\rangle, e_{I(Y)}\right)$ is a dual residuated connection.

(3) $\left(e_{I(X)},\left[R^{-1}\right]^{c},\left[S^{-1}\right]^{c}, e_{I(Y)}\right)$ is not a Galois connection because $\left[S^{-1}\right]\left(\{y\}^{c}\right)=\{a, c, d\} \notin I(X)$.

(4) $\left(e_{I(X)},\left[\left\langle R^{-1}\right\rangle\right],\left[\left\langle S^{-1}\right\rangle\right], e_{I(Y)}\right)$ is not a dual Galois connection because $\left[\left\langle S^{-1}\right\rangle\right](\{y\})=\{a\} \notin I(X)$.

(5) $\left(e_{I(X)},\left\langle R^{-1}\right\rangle^{c},\left\langle S^{-1}\right\rangle^{c}, e_{I(Y)}\right)$ is not a dual Galois connection because $\left\langle R^{-1}\right\rangle^{c}(\{b, d\})=\{x, z\} \notin I(Y)$.

(6) $\left(e_{I(X)},\left[\left[R^{-1}\right]\right],\left[\left[S^{-1}\right]\right], e_{I(Y)}\right)$ is not a Galois connection because $\left[\left[R^{-1}\right]\right](\{c, d\})=\{x, z\} \notin I(Y)$.

(7) $\left(e_{I(X)},\left[\left(R^{c}\right)^{-1}\right],\left\langle\left(S^{c}\right)^{-1}\right\rangle, e_{I(Y)}\right)$ is not a dual residuated connection because $\left[\left(R^{c}\right)^{-1}\right](\{d\})=\{x\} \notin I(Y)$.

\section{References}

[1] R. Bělohlávek, Lattices of fixed points of Galois connections, Math. Logic Quart. 47 (2001), 111-116.

[2] G. Georgescu, A. Popescue, Non-dual fuzzy connections, Arch. Math. Logic 43 (2004), 1009-1039.

[3] J. Järvinen, M. Kondo, J. Kortelainen,, Logics from Galois connections, Internat. J. Approx. Reason. 49 (2008), 595-606.

[4] Y.C. Kim, Y.S. Kim, Relations and upper sets on partially ordered sets, Int. Math. Forum 6 (19) (2011), 899-908.

[5] Ewa. Orlowska, I. Rewitzky, Algebras for Galois-style connections and their discrete duality, Fuzzy Sets and Systems 161 (2010), 1325-1342.

[6] Z. Pawlak, Rough sets, Int. J. Comput. Inf. Sci. 11 (1982), 341-356.

[7] G. Qi, W. Liu, Rough operations on Boolean algebras, Inform Sciences $\mathbf{1 7 3}$ (2005), 49-63.

[8] R. Wille, Restructuring lattice theory; an approach based on hierarchies of concept, in: 1. Rival(Ed.), Ordered Sets, Reidel, Dordrecht, Boston, 1982.

Department of Mathematics

Natural Science

Gangneung-Wonju National University

Gangneung, Gangwondo

210-702, Korea 
E-mail: yck@gwnu.ac.kr

Department of Mathematics

Natural Science

Gangneung-Wonju National University

Gangneung, Gangwondo

210-702, Korea

E-mail: jmko@gwnu.ac.kr 